\title{
Flocculus Herniation and Hearing Disturbance Induced by Remote Tentorial Meningioma: A Case Report
}

\author{
Yuhei Michiwaki, ${ }^{1,2}$ Takuya Inoue, ${ }^{2}$ and Yasutoshi Kai ${ }^{2,3}$
}

Tentorial meningiomas, not involving the cerebellopontine angle cistern or cranial nerves, rarely cause symptoms of cranial nerve disturbance. We report a case of a patient with a paramedian tentorial meningioma who presented with hearing loss as a result of indirect compression of the vestibulocochlear nerve by the herniated cerebellar flocculus into the internal acoustic meatus. A 50-year-old woman had presented with hearing loss in her right ear. Magnetic resonance imaging (MRI) revealed a large tentorial meningioma in the right posterior cranial fossa. Constructive interference in steadystate (CISS) imaging demonstrated a non-enhanced solid structure at the ipsilateral cerebellopontine angle cistern and internal acoustic meatus. During surgery, after resection of the tumor, the herniated cerebellar flocculus into the internal auditory canal was observed at the ipsilateral cerebellopontine angle. MRI obtained following meningioma resection demonstrated the herniated flocculus regressing from the fundus of the internal acoustic meatus to the cerebellopontine angle cistern, and her hearing was improved as a result of decompression. This is a rare case report of flocculus herniation caused by remote tentorial meningioma. Patients with paramedian tentorial meningiomas rarely present with hearing loss. In these cases, the causes of the hearing loss (microvascular compression, transformed brainstem, and venous circulation disorders) have been described in the literature. In this report, a new mechanism became evident: a herniated flocculus into the internal acoustic meatus by a tumor can cause hearing loss. MRI, particularly CISS imaging, can clearly show the flocculus during the entire clinical course.

Keywords: tentorial meningioma, flocculus, herniation, internal acoustic meatus, hearing disturbance

\section{Introduction}

Tentorial meningiomas account for $2-5 \%$ of all intracranial meningiomas. ${ }^{1,2)}$ Tentorial meningiomas arise from the tentorium cerebelli and are classified by anatomical location

${ }^{1}$ Department of Neurosurgery, Graduate School of Medical Sciences, Kyushu University, Fukuoka, Fukuoka, Japan

${ }^{2}$ Department of Neurosurgery, Kyushu Central Hospital of the Mutual Aid Association of Public School Teachers, Fukuoka, Fukuoka, Japan

${ }^{3}$ Department of Neurosurgery, lizuka Hospital, lizuka, Fukuoka, Japan

Received: March 8, 2018; Accepted: May 1, 2018

Online September 20, 2018

Copyright $₫ 2018$ by The Japan Neurosurgical Society

This work is licensed under a Creative Commons AttributionNonCommercial-NoDerivatives International License. as follows: incisural (anterior or middle), falcotentorial, paramedian, peritorcular, and lateral types. ${ }^{3,4)}$

Patients with incisural and lateral types of tentorial meningiomas often present with cranial nerve symptoms because the tumors grow near the cranial nerves. Patients with paramedian and peritorcular types of tentorial meningiomas, however, present with headaches or vomiting (both caused by elevated intracranial pressure) or cerebellar ataxia, and rarely cause cranial nerve symptoms. ${ }^{3)}$

We report a case of a patient with a remote paramedian tentorial meningioma who presented with hearing loss due to herniation of the cerebellar flocculus into the internal acoustic meatus as a result of tumor compression.

\section{Case Report}

History: A 50-year-old woman had occasionally presented unsteady gait a few years ago and presented with hearing loss in her right ear. She consulted an otolaryngologist and was diagnosed with right sudden deafness. She was recommended to have detailed physical examination and came to our hospital where magnetic resonance imaging (MRI) revealed a large brain tumor in the right posterior cranial fossa with concomitant hydrocephalus.

Preoperative examination: The neurological examination performed upon admission was unremarkable, except for mild right hearing loss, particularly in the high sound areas. A pure tone audiogram (PTA) revealed hearing ability of $25.0 \mathrm{~dB}$ (categorized in mild hearing loss) in the right ear and $7.5 \mathrm{~dB}$ (categorized in normal hearing) in the left ear by the one-fourth method (Fig. 1). She did not have paresis, sensory disturbances, or limb ataxia; her tandem gait, however, was slightly ataxic. T1-weighted image (T1WI) with contrast enhancement revealed a $54 \times 55 \times 58 \mathrm{~mm}^{3}$ strongly enhanced tumor in the right posterior cranial fossa. The tumor was diagnosed as a paramedian tentorial meningioma (Fig. 2A). T1WI and constructive interference in steady-state (CISS) imaging demonstrated a non-enhanced solid structure at the ipsilateral cerebellopontine angle cistern and internal acoustic meatus (Figs. 2B and 2C). Angiographically, the meningioma was fed by the occipital and middle meningeal arteries. The ipsilateral transverse sinus was occluded by the tumor.

Surgery: The patient underwent surgery in the prone position with a right-sided combined occipital/suboccipital craniotomy. Simpson grade II resection was achieved comprising a complete resection of the tumor involving the tentorium 
with focal coagulation of dural attachment of the confluence of sinus. After resection of the tumor, the herniated cerebellar flocculus into the internal auditory canal was observed at the ipsilateral cerebellopontine angle (Fig. 3A). The surgeon did not remove the herniated flocculus due to the principle that it should be better to leave the normal structure as it is.

Postoperative course: Histopathology revealed findings typical of a transitional meningioma. The patient did not present with any new neurological deficits other than the mild dizziness, and a PTA examined 2 weeks following the surgery revealed improvement in her hearing ability of 13.8 $\mathrm{dB}$ (categorized in normal hearing) in the right ear (Fig. 4A). She was discharged to her home 20 days after the surgery (Karnofsky performance status 90\%). A PTA obtained one

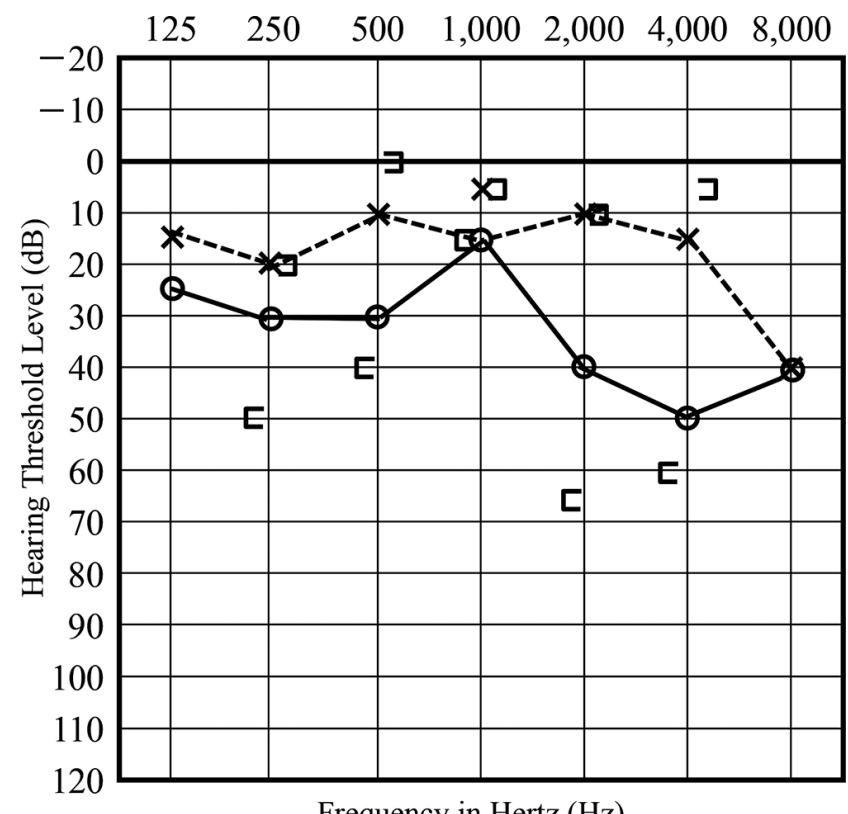

Frequency in $\operatorname{Hertz}(\mathrm{Hz})$

Fig. 1 Preoperative pure tone audiogram revealed a sensorineural hearing loss with pure tone threshold of $28.3 \mathrm{~dB}$ in the right ear by the one-fourth method. year following the surgery demonstrated slight improvement in her hearing ability of $12.5 \mathrm{~dB}$ (Fig. 4B).

Postoperative imaging: Postoperative MRI demonstrated complete resection of the meningioma (Fig. 3B). As regards the flocculus in the internal acoustic meatus, MRI obtained the day following the surgery demonstrated the flocculus regressing from the fundus of the internal acoustic meatus to the cerebellopontine angle cistern (Fig. 5A). MRI obtained 3 months after the surgery revealed that the flocculus was seen near the internal auditory canal (Fig. 5B); 6 months after the surgery, no structure existed in the internal acoustic meatus, and the flocculus was out of the internal auditory canal (Fig. 5C).

\section{Discussion}

In the tentorial meningiomas, the headache is the most frequent symptom and is followed by limb and gait ataxia or nausea and vomiting. These symptoms are caused by the elevated intracranial pressure or cerebellar impairment. They rarely present with the symptoms of cranial nerve disturbance, particularly paramedian type of tentorial meningiomas that are without any contact with the cranial nerves. ${ }^{1,3)}$ In our case, a large paramedian tentorial meningioma remotely located from any cranial nerves induced hearing loss as a result of disturbance of vestibulocochlear nerve due to the flocculus herniation into the internal acoustic meatus.

Anatomically, the flocculus is located posterior to the facial and vestibulocochlear nerves and caudal side of the internal auditory canal. ${ }^{5)}$ There is cerebrospinal fluid space between the flocculus and the internal auditory canal; therefore, any normal tissue cannot invaginate into the internal auditory canal in the normal state. However, a large mass, such as a tumor or hemorrhage, can compress the cerebellum dramatically to the anterior (ventral) side, the flocculus may be compressed into the internal auditory canal indirectly, and flocculus herniation may be caused by indirect compression. And the decompression of the cerebellum after surgery induced the recovery from the flocculus herniation in our case. The rounded structure and mild mobility of the flocculus may be responsible for this rare situation.
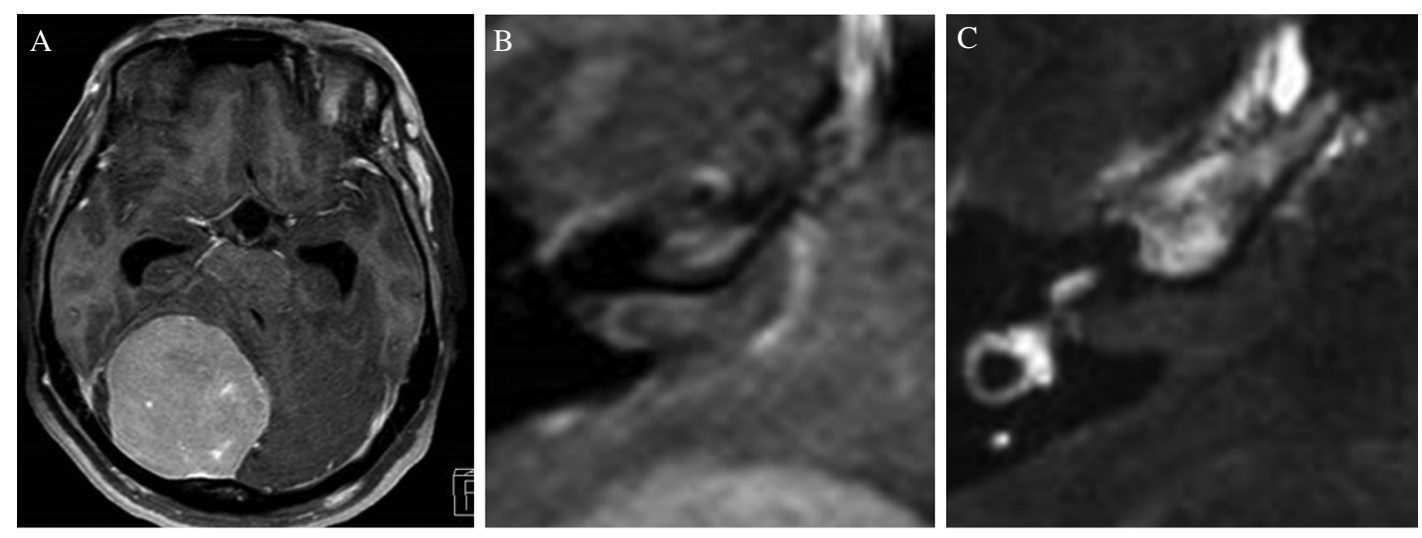

Fig. 2 (A) Preoperative T1WI with contrast enhancement showing a large tentorial meningioma and coexisting hydrocephalus. (B) Preoperative T1WI with contrast enhancement showing a non-enhanced solid structure at the ipsilateral cerebellopontine angle cistern and internal acoustic meatus. (C) MR CISS imaging obtained at the same time and from the same slice. 


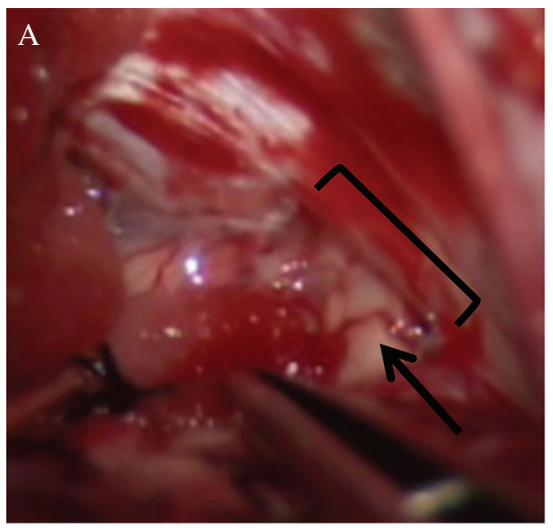

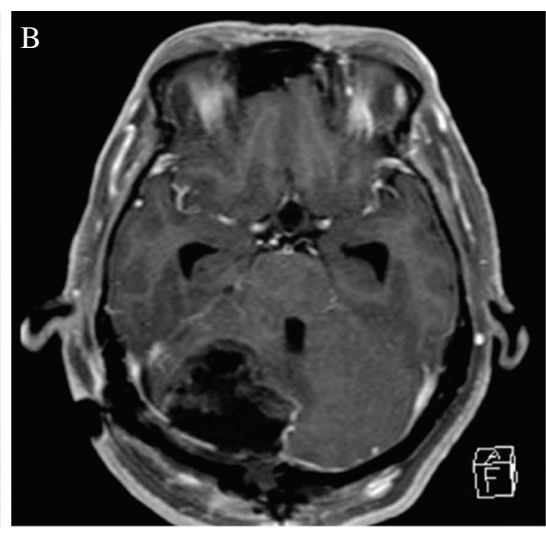

Fig. 3 (A) At the ipsilateral cerebellopontine angle cistern, the cerebellar flocculus (arrow) had herniated into the internal auditory canal. (B) Postoperative T1WI with contrast enhancement showing complete resection of the meningioma (Simpson Grade II).
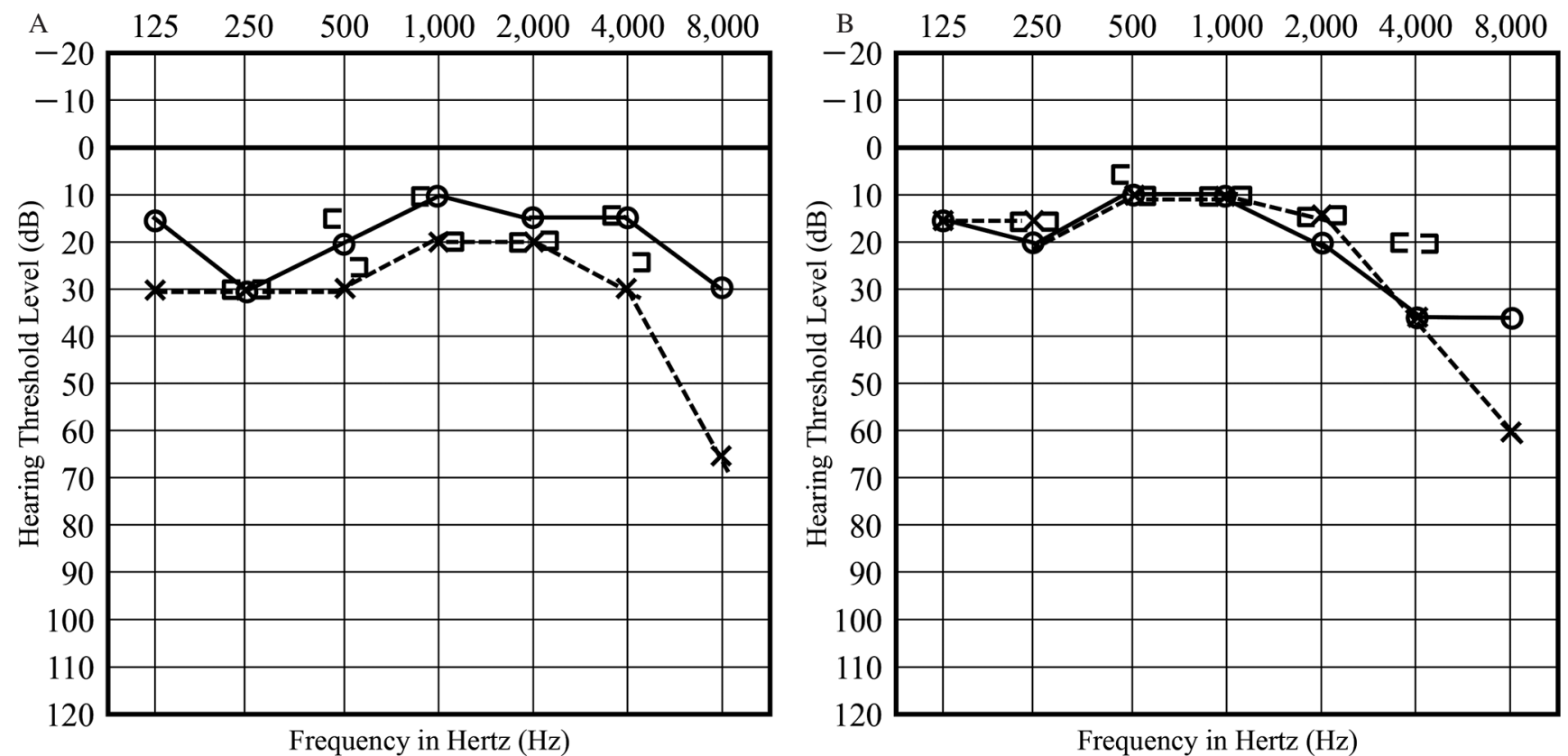

Fig. 4 (A) Postoperative pure tone audiogram (PTA) examined 2 weeks following the surgery revealed improvement of hearing with pure tone threshold of $15.0 \mathrm{~dB}$ in the right ear by the one-fourth method. (B) A PTA obtained 1 year following the surgery demonstrated slight improvement in her hearing with pure tone threshold of $13.3 \mathrm{~dB}$ in the right ear by the one-fourth method.

Tentorial meningiomas, which are remotely located from any of the cranial nerves, rarely cause cranial nerve symptoms; only a few cases have been reported. Ridder et al. reported a patient with a tentorial meningioma (much smaller than in our case) who presented with sensorineural deafness. ${ }^{6)}$ The meningioma had grown far away from vestibulocochlear nerve and acoustic radiations and hearing improved after resection of the meningioma. They hypothesized that the deafness resulted from neurovascular compression of the vestibulocochlear nerve caused by the growing meningioma. They discussed the possibility that the compression of the brainstem could cause deafness; however, the unilateral deafness seen in their case was extremely rare because the auditory tract follows a bilateral trajectory.

Some cases have reported that similar remote tentorial meningiomas caused trigeminal neuralgia or hemifacial spasm. ${ }^{7-10)}$ Ogasawara et al. discussed that these phenomena may result from both compression and displacement of the brainstem and secondary neurovascular compression of the trigeminal nerve by the tumor. They termed these mechanisms as "remote effects." ") In addition to these mechanisms, Cancelli et al. hypothesized that venous circulation disorders can contribute to these phenomena. ${ }^{10)}$

While we cannot completely exclude these factors, such as neurovascular compression, disturbance of venous circulation, or elevated intracranial pressure were responsible for hearing disturbance in our case, it seems to be no doubt that flocculus herniation into the internal acoustic meatus was one of the causes of hearing disturbance. Considering that the degree of hearing improvement following the surgery was less than $15 \mathrm{~dB}$, it cannot be excluded that it was within the physiological variation or error range. If the surgeon removed the herniated flocculus, and intraoperative auditory brainstem response recovered at the same time during surgery, it might have been more obvious that the flocculus herniation caused hearing disturbance. In our 

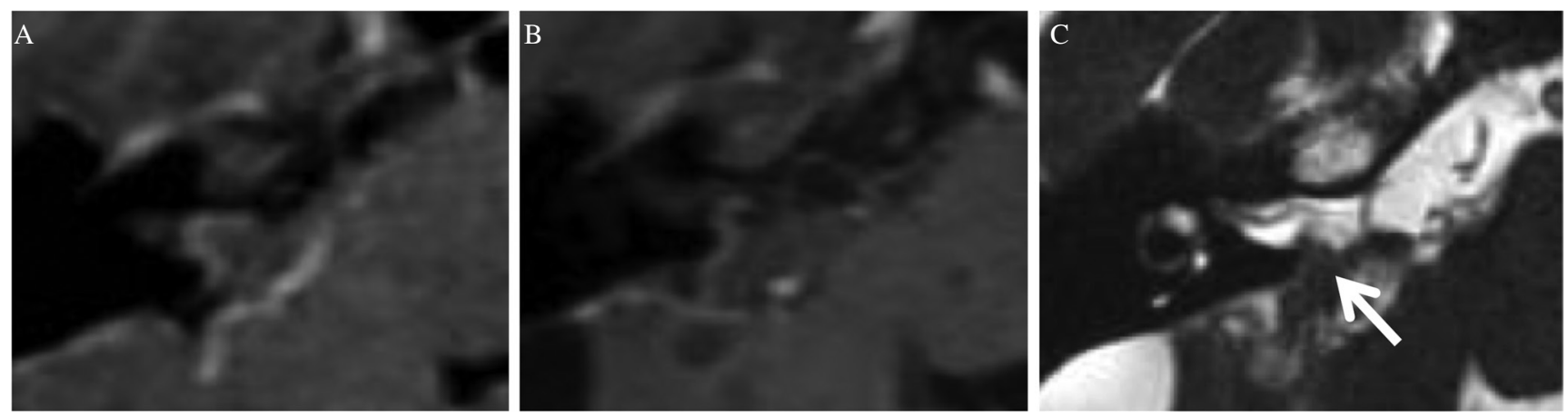

Fig. 5 (A) T1-weighted image (T1WI) with contrast enhancement obtained the next day after surgery showing that herniated flocculus regressed to the cerebellopontine angle cistern from the fundus of internal acoustic meatus. (B) T1WI with contrast enhancement obtained 3 months after the surgery showing that the herniated flocculus almost disappeared inside of the internal acoustic meatus. (C) MR CISS imaging performed six months after the surgery revealing no structure inside of the internal acoustic meatus and the flocculus (arrow) was evident at the cerebellopontine angle cistern continuous to the internal auditory canal.

case, the hearing ability improved following the surgery still the flocculus was detected in the internal acoustic meatus in postoperative MRI. This result can be explained by the decrease in pressure to the flocculus due to meningioma resection. The occurrence of hearing loss by the herniated flocculus into the internal acoustic meatus has not been described in the literature ${ }^{11)}$; to our knowledge, this is a rare case report.

\section{Conclusion}

Patients with paramedian tentorial meningiomas rarely present with hearing loss. In these cases, the causes of the hearing loss (microvascular compression, transformed brainstem, and venous circulation disorders) have been described in the literature. In this report, a new mechanism became evident: a herniated flocculus into the internal acoustic meatus by a tumor can cause hearing loss. MRI, particularly CISS imaging, can clearly show the flocculus during the entire clinical course.

\section{Conflict of Interest Disclosure}

All authors have no conflict of interest.

\section{References}

1) Gökalp HZ, Arasil E, Erdogan A, Egemen N, Deda H, Cerçi A: Tentorial meningiomas. Neurosurgery 36: 46-51, 1995

2) Bret P, Guyotat J, Madarassy G, Ricci AC, Signorelli F: Tentorial meningiomas. Report on twenty-seven cases. Acta Neurochir (Wien) 142: 513-526, 2000

3) Bassiouni H, Hunold A, Asgari S, Stolke D: Tentorial meningiomas: clinical results in 81 patients treated microsurgically. Neurosurgery 55: 108-116; discussion 116-118, 2004

4) Yasargil MG. Microneurosurgery of CNS tumors. Vol. 4B. New York, Georg Thieme Verlag; 1996. pp. 134-165.

5) Rhoton AL Jr: The cerebellopontine angle and posterior fossa cranial nerves by the retrosigmoid approach. Neurosurgery 47: S93-S129, 2000

6) De Ridder D, Menovsky T, Van Lear C, Van de Heyning P: Remote tentorium meningioma causing sudden sensorineural deafness. Surg Neurol 70: 312-317; discussion 318, 2008

7) Park H, Hwang SC, Kim BT, Shin WH: Hemifacial spasm caused by a huge tentorial meningioma. J Korean Neurosurg Soc 46: 269-272, 2009

8) Ogasawara H, Oki S, Kohno H, Hibino S, Ito Y: Tentorial meningioma and painful tic convulsif. Case report. J Neurosurg 82: 895-897, 1995

9) Perrini P, Rasile F, Laggate J: Trigeminal neuralgia as initial symptom of paramedian tentorial meningioma. Neurol Sci 30: 81-83, 2009

10) Cancelli I, Cecotti L, Valentinis L, Bergonzi P, Gigli GL: Hemifacial spasm due to a tentorial paramedian meningioma: a case report. Neurol Sci 26: 46-49, 2005

11) Verbist BM: Imaging of sensorineural hearing loss: a pattern-based approach to diseases of the inner ear and cerebellopontine angle. Insights Imaging 3: 139-153, 2012

Corresponding author:

Yuhei Michiwaki, MD, Department of Neurosurgery, Graduate School of Medical Sciences, Kyushu University, 3-1-1 Maidashi, Higashi-Ku, Fukuoka, Fukuoka 812-8582, Japan. $\triangle$ wayside.bamboo@gmail.com 